\title{
Pressure Pulsation in Kaplan Turbines: Prototype-CFD Comparison
}

\author{
A Rivetti ${ }^{1}$, C Lucino1 ${ }^{1}$, S Liscia ${ }^{1}$, D Muguerza ${ }^{2}$, F Avellan ${ }^{3}$ \\ ${ }^{1}$ Laboratorio de Hidromecánica, Facultad de Ingeniería, Universidad Nacional de La \\ Plata, 47 N $^{\circ}$ 200, La Plata, Argentina. \\ ${ }^{2}$ Consejo de la Entidad Binacional Yacyretá (EBY), Paraguay-Argentina, La Rioja \\ 1640, Posadas, CP 3300, Misiones \\ ${ }^{3}$ Laboratory for Hydraulic Machines, École Polytechnique Fédérale de Lausanne \\ (EPFL), Avenue de Cour 33bis, CH-1007, Lausanne, Switzerland.
}

E-mail: arturorivetti@gmail.com, clucino@ing.unlp.edu.ar, soliscia@ing.unlp.edu.ar, ingeniero.muguerza@gmail.com, francois.avellan@epfl.ch.

\begin{abstract}
Pressure pulsation phenomena in a large Kaplan turbine are investigated by means of numerical simulations (CFD) and prototype measurements in order to study the dynamic behavior of flow due to the blade passage and its interaction with other components of the turbine. Numerical simulations are performed with the commercial software Ansys CFX code, solving the incompressible Unsteady Reynolds-Averaged-Navier Stokes equations under a finite volume scheme. The computational domain involves the entire machine at prototype scale. Special care is taken in the discretization of the wicket gate overhang and runner blade gap. Prototype measurements are performed using pressure transducers at different locations among the wicket gate outlet and the draft tube inlet. Then, CFD results are compared with temporary signals of prototype measurements at identical locations to validate the numerical model.

A detailed analysis was focused on the tip gap flow and the pressure field at the discharge ring. From a rotating reference frame perspective, it is found that the mean pressure fluctuates accordingly the wicket gate passage.

Moreover, in prototype measurements the pressure frequency that reveals the presence of modulated cavitation at the discharge ring is distinguished, as also verified from the shape of erosion patches in concordance with the number of wicket gates.
\end{abstract}

Keywords: Kaplan Turbine, CFD, pressure pulsation, prototype measurements.

\section{Introduction}

During the last decades, Kaplan power output has increased continuously and larger diameters were designed accordingly. But only since recently can certain features (e.g. tip vortices development or rotor-stator interaction phenomena) be studied at design stage by means of modeling techniques. As a result of the advance of CFD models, a more insightful analysis can be made of these particular flow features. Then, experimental and prototype data are of great importance in order to validate simulation results.

Extensive literature is available about phenomena related to dynamic behavior of the pressure pulsation and RSI (Rotor-Stator Interaction) in Francis and Pump turbines [1, 2 (and reference 
therein)]. However, such state of knowledge has not been reached in Kaplan turbines yet. Shuhong [3] achieved good results in the comparison of pressure pulsations obtained by unsteady CFD and experimental measurements in Kaplan physical models. In addition, Nennemann [4] studied the influence of non-uniformity of the flow at the guide vanes outlet and its interaction with the tip gap vortex using CFD. Also, by analyzing the interaction of wicket gate and runner pressure fields he could explain the existence of a characteristic frequency that evidences the presence of cavitation at circumferential locations corresponding to the number of wicket gates. Gagnon [5,6] analyzed the potential of unsteady CFD to predict pressure pulsations and rotor-stator interaction obtaining good results. Nilsson [7] studied the development of the tip clearance flow in a Kaplan turbine and found a representation of the tip gap vortex using a relatively coarse mesh and the k- $\varepsilon$ turbulence model.

In the present work, firstly the case of study of a large Kaplan turbine at prototype scale operating at the minimum guaranteed head is introduced. Then, an overview of the numerical simulation implemented is summarized, including the numerical scheme, spatial discretization, boundary conditions and convergence history. Results of the numerical simulation are validated against prototype measurements of pressure located at several points in the discharge ring wall. Finally, a qualitative and quantitative analysis of numerical results is performed.

\section{Case study}

An entire Kaplan turbine is studied in this work. The stator is composed of 24 guide and stay vanes and the runner has 5 blades. The semi-spiral casing is divided in three channels by two vanes, the same as the draft tube. This presents a slight asymmetry in order to be aligned with the semi-spiral casing (Fig. 1).

The operating point simulated is indicated over on the efficiency chart (Fig. 2) and it corresponds to a wicket gate opening of $86 \%$ at the minimum guaranteed head of the machine

As can be seen on the chart, during prototype measurements, several operating points corresponding to a minimum wicket gate opening to a full gate opening were performed. The locations of pressure sensors over the prototype machine are shown in Fig.3. All the operating points measured correspond to on-cam combination.

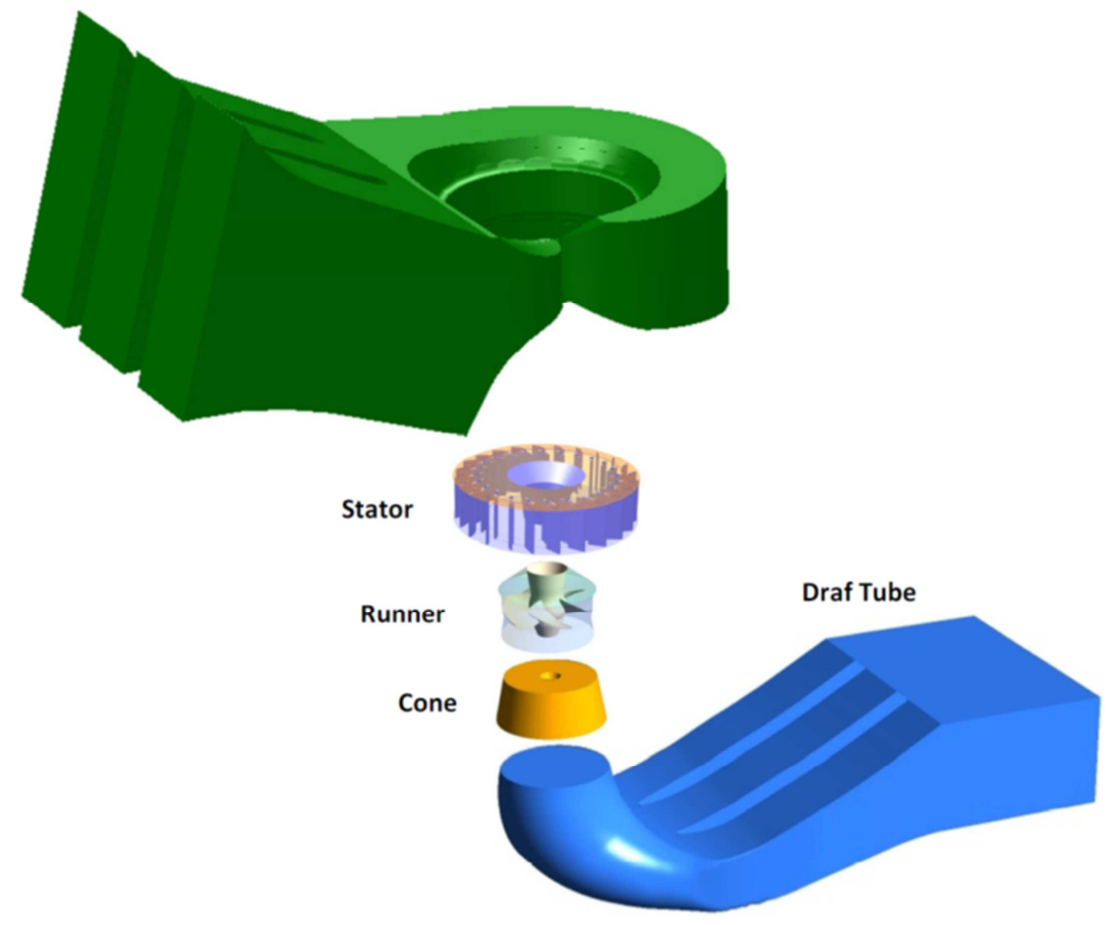

Fig. 1 Computational Domain. 


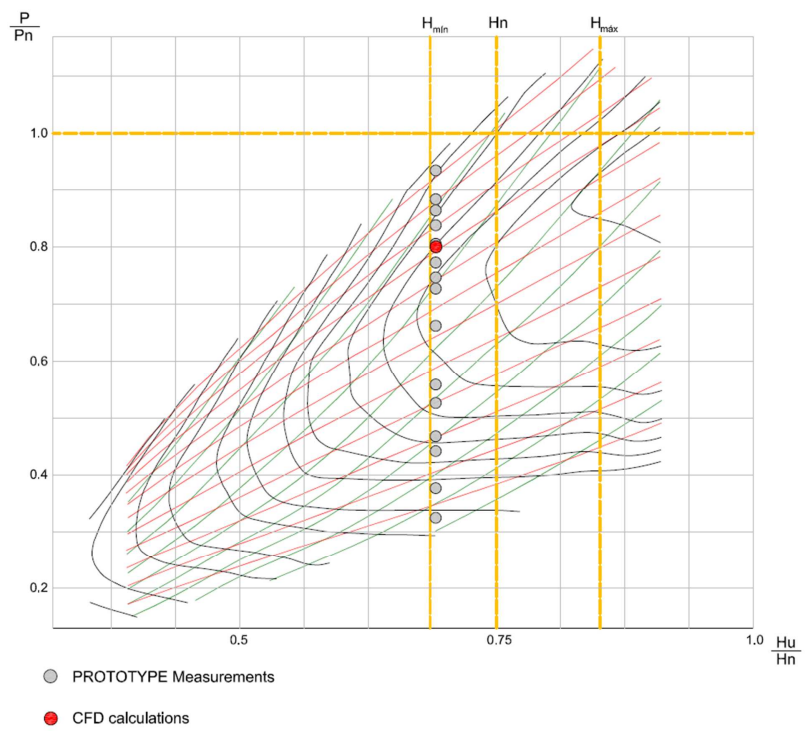

Fig. 2 Point measured and simulated over the discharge hill.

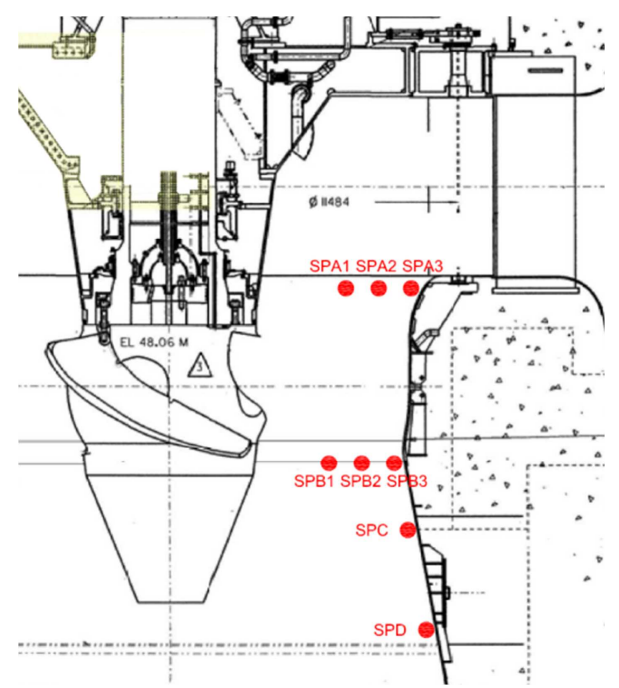

Fig. 3 Pressure transducer locations.

\section{Numerical simulation}

The numerical simulation is performed using the commercial code ANSYS CFX 13.0, that solves the Unsteady Reynolds Average Navier Stokes equations (URANS). The turbulence model used to close the system of equations is the RNG k-E, and the advection scheme imposed is of the second order, both in space and time. First a steady-state flow simulation is performed in order to use it as the initial flow conditions. In this case, a frozen rotor interface is used to make the stationary and rotating domains compatible. For the transient case, a transient-rotor-stator interface is used with a time step corresponding to $1^{\circ}$ of rotation, in order to capture the dynamic behavior of the machine. Convergence is reached for root mean square (RMS) average of normalized velocities and pressure residuals between $10^{-5}$ and $10^{-6}$ in all cases.

A hybrid mesh is generated in order to discretize the domain. For the semiespiral casing, a tetrahedral non-structured mesh of $2,000 \mathrm{k}$ elements is used. The rest of the components are discretized into hexahedral structured elements, due to their better performance when simulating secondary flows and a more efficient use of the computational resources during simulation. The meshing quality is analyzed considering the sensitivity of the torque due to the $y+$ value. Several mesh sizes are analyzed in order to ensure the independence of results. In addition, special attention is paid to the distributor gap caused by the blade overhanging (Fig. 4) in order to simulate the potential influence in the tip gap vortex of the runner blade. Table 1 summarizes the characteristics of the mesh for every part of the computing domain.

Table 1 Spatial discretization.

\begin{tabular}{ccccc}
\hline Domain & Type of Domain & Type of mesh & Number of elements & y+ (average) \\
\hline Semi-spiral casing & Stationary & Tetrahedral & $2.2 * 10^{6}$ & 182 \\
Stator & Rotating & Hexahedral & $11.5 * 10^{6}$ & 47 \\
Runner & Stationary & Hexahedral & $6.7 * 10^{6}$ & 112 \\
Cone & Stationary & Hexahedral & $0.6^{*} 10^{6}$ & 126 \\
Draft Tube & Stationary & Hexahedral & $2.4 * 10^{6}$ & 143 \\
Gap & Stationary & Hexahedral & $0.2 * 10^{6}$ & 108 \\
All Domains & & Hybrid & $\mathbf{2 3 . 7 * 1 0 ^ { 6 }}$ & $\mathbf{9 1}$ \\
\hline
\end{tabular}




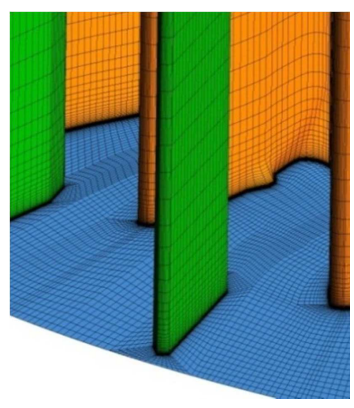

(a)

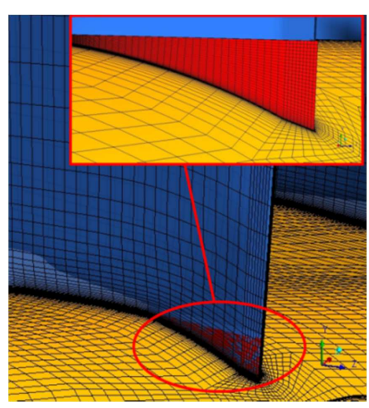

(b)

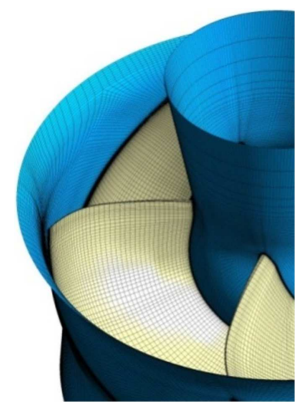

(c)

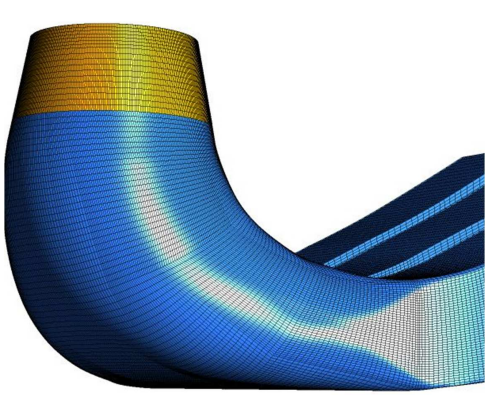

(d)

Fig. 4. Hexahedral structured mesh; (a) The stator mesh with the stay vanes (green) and wicket gates (orange); (b) Detail of the gap domain (red); (c) The runner; (d) The draft tube.

Boundary conditions are chosen so that they are able to reproduce the same operating point as that studied for prototype measurements. The operating information obtained from measurements consists of the power output, the net head, the tail water level, and the positions of the guide vanes and runner blades. The flow rate is obtained from the hill chart and used to compute the average velocity at the inlet section.

A normal velocity is selected at the inlet, and an average static pressure for the outlet. The value for the pressure outlet is set to zero, and a hydrostatical reference level $(\Delta z)$ is set to have the same pressure profile as prototype (Eq. 1). A value of $1 \mathrm{~atm}$ is adopted as the reference pressure. The general grid interface (GGI) is used in order to materialize the connections between adjacent domains. The no-slip condition is imposed on all the solid surfaces and a counter-rotating wall configuration is set for the surfaces composing the rotating domain that are not rotating.

$$
p_{a b s}=p_{\text {ref }}+p_{\text {est }}+\rho g \Delta z \quad \text { Eq. } 1
$$

The net head $(\mathrm{H})$, the flow rate (Q) and the power output (Pot) are monitored during calculations. Four hundred iterations were necessary to obtain constants values for these parameters. Once this condition is reached, transient calculations were carried out, requiring 1,500 time steps that correspond to four runner passages. For every time step, ten internal coefficient loops are selected, and the pressure is monitored at the same spatial locations as pressure transducers at the prototype. For the last two impeller rotations, the pressure reveals a periodic fluctuation and constants mean values. This data is used to do the comparison between prototype measurements.

\section{Results}

\subsection{CFD validation}

Several monitoring points are placed at the same locations as the pressure sensors in the prototype so that both signals could be compared during the transient simulation. The temporal pressure signal for both prototype and CFD simulation is plotted in Fig. 5 as a function of a dimensionless pressure coefficient, Cp (Eq. 2), and $\mathrm{n}$ as the time variable. A good agreement was found in most cases -both the magnitude of pressure pulsation and the shape of the signal as simulated are clearly similar to those obtained experimentally. However, the pressure pulsation due to the blade passage was overestimated by the numerical model at the location of sensor D. Furthermore, high frequencies, as captured by pressure sensors, cannot possibly be obtained by the numerical simulation due the time step used. 


$$
C p=\frac{p_{a b s}-\bar{p}_{a b s}}{\rho E}
$$

Eq. 2
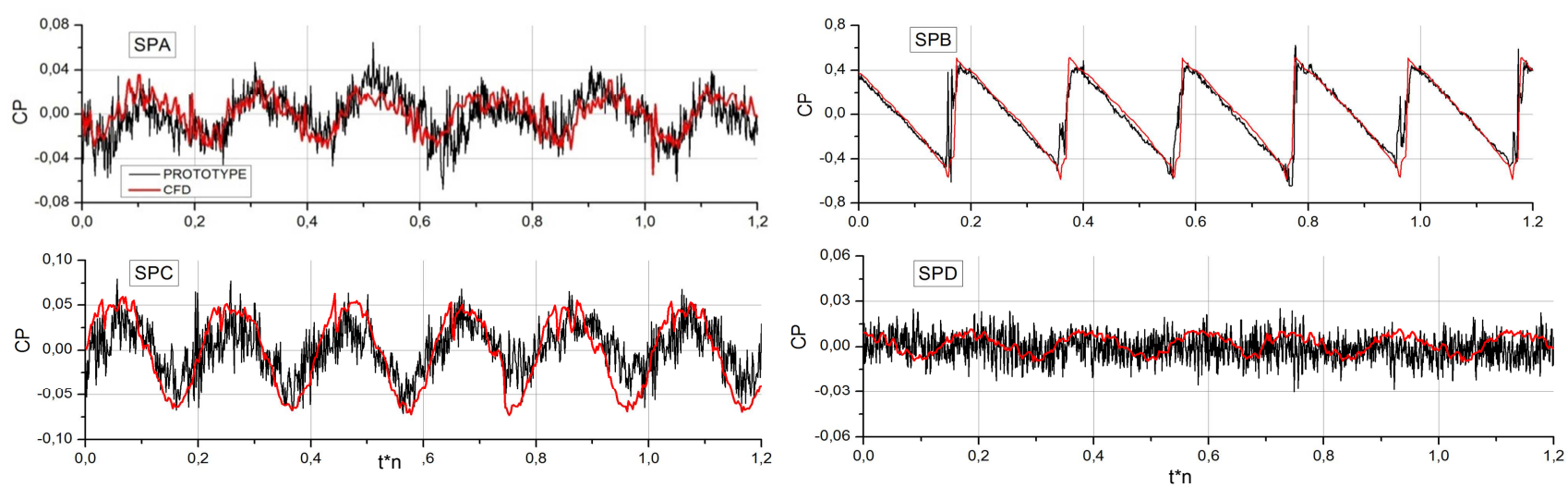

Fig. 5 Numerical versus prototype measurements temporary signals.

The results of Fast Fourier Transform (FFT) performed on the pressure signals of both prototype measurements and numerical simulations are shown in Fig.6. There is good agreement in blade passage amplitude (5n) for all cases with the exception of sensor D. As well as in the temporal signal, the amplitude of blade passage is overestimated. Also for the sensor D, the frequency of $25 \mathrm{n}$ is not predicted by the numerical simulation. This frequency is related with the modulation of stay vanes and rotor pressure fields [4] that reveals the presence of localized cavitation over the discharge ring. In sensors $\mathrm{A}, \mathrm{B}, \mathrm{C}$ this frequency is also present but is negligible compared against blade runner passage and their harmonics. Further research is needed in this area.
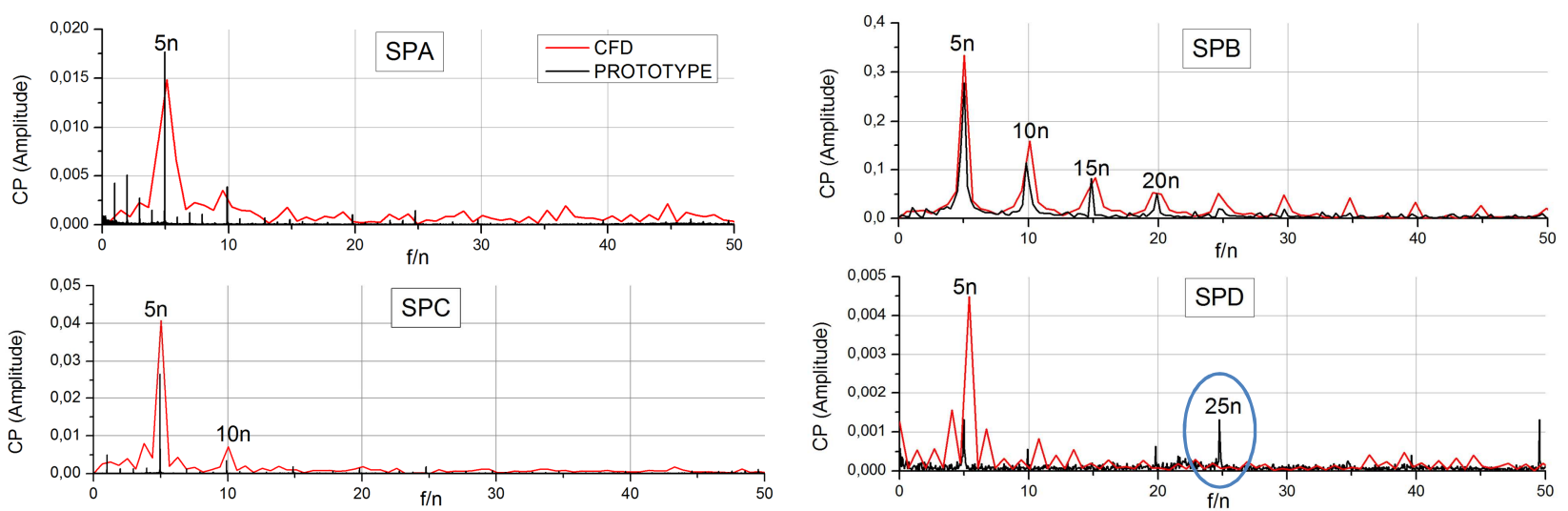

Fig. 6 Numerical versus prototype measurements spectra.

\subsection{Qualitative analysis}

A view of the suction side of the blade (Fig. 7a), where an iso-surface of pressure is plotted, shows the development of the tip gap vortex through the blade edge. The pressure on the discharge ring wall shows the contact zone of the tip gap vortex against the wall (Fig. 7b).

The absolute pressure distribution for three planes located at 75,85 and $95 \%$ of the chord length is shown in Fig.8. Two zones of low pressure can be identified, the first one induced by a local acceleration of the flow entering into the gap, and the second one corresponding to the tip gap vortex. It can be seen that the vortex strength increases as it evolves downstream while making contact against 
the wall (Zone A, Fig. 7 and 8). The flow pattern at the gap generated by the wicket gate overhang is shown in Fig. 9. Two vortices are produced by the shear velocity of the free stream flow. These flow structures are advected from the wicket gate downstream by the mean flow which modifies their initial trajectory and intensity. The streamlines that arise from the vortex structure show this phenomenon (Fig. 9b). This particularity increases the non-uniformity of the pressure that interacts with the flow under the runner blade.

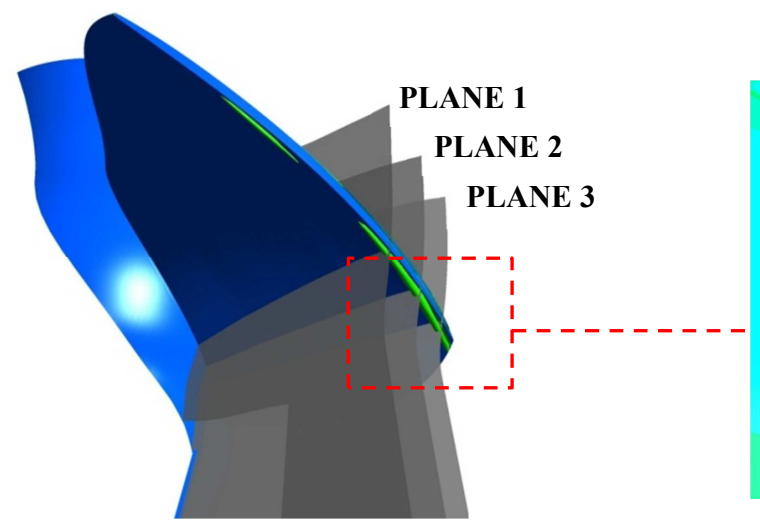

(a)

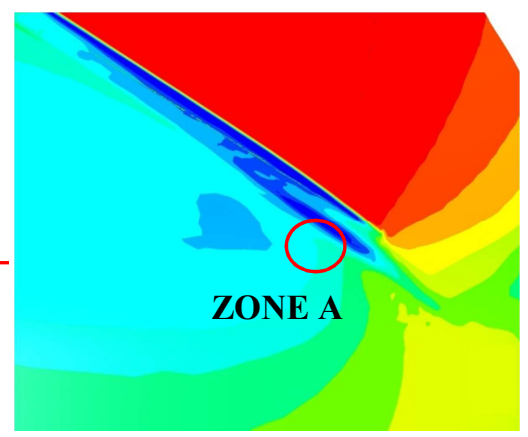

(b)

Fig. 7 (a) Development of the tip gap vortex using an iso-surface of constant pressure; (b) Pressure color map where the contact of the tip vortex against the discharge ring wall is indicated.

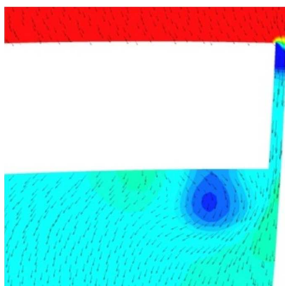

Plane 1

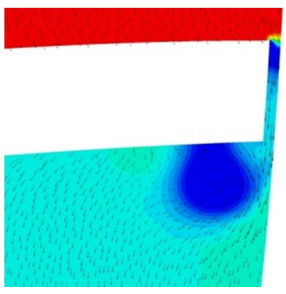

Plane 2

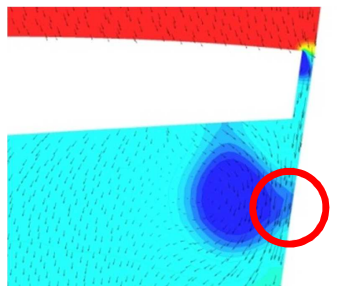

Plane 3

ZONE A

Fig. 8 Pressure distribution for planes 1, 2 and 3 indicated on Fig. 7a. In plane 3, the contact between the tip vortex and the discharge ring wall is indicated.

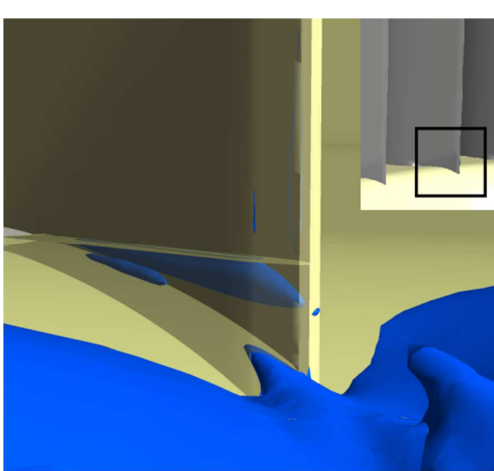

(a)

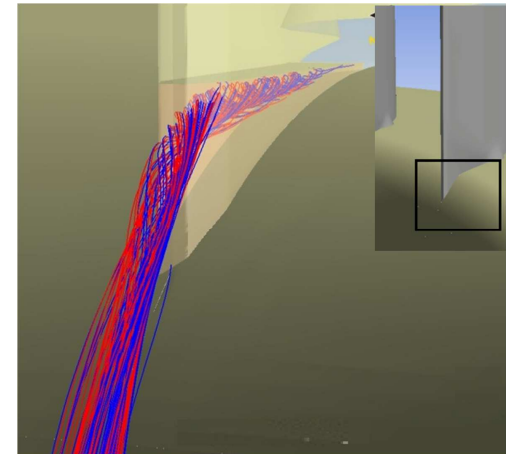

(b)

Fig. 9 Flow through wicket gate overhang gap. (a) Iso-surface of pressure showing the two vortexes generated in the gap cavity. (b) Streamlines starting from the vortex surface. 


\subsection{RSI interaction over the blade suction side.}

To evaluate the influence of the non-uniform flow at the distributor outlet with the blade passage, four pressure points were placed in the spanwise direction (Fig. 10). The distance from these points to the wall is four times the gap size (4e) in correspondence with the tip vortex core. This analysis was carried out considering a frame of reference that is rotating with the runner velocity. Temporary signals (Fig. 11a) give a clear indication that the pressure fluctuates with the wicket gate passage. The maximum fluctuation amplitude is found at point one and it decreases along the spanwise direction reaching the minimum amplitude at point three. For point four it is seen an increment in the amplitude in comparison with point three. This reveals the influence of the wicket gate over the pressure field in the discharge ring that generates a discrete pattern of cavitation in concordance with the number of wicket gates. In the FFT analysis this phenomenon is confirmed (Fig.11b). The frequency of wicket gate passage is $24 \mathrm{n}$. Also, the harmonic frequencies are present $(48,72,96$ and $120 \mathrm{n})$.

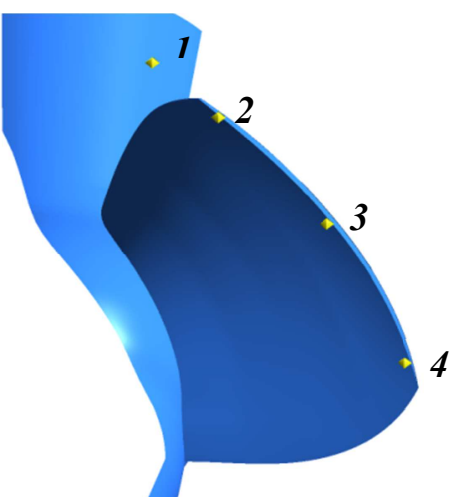

(a)

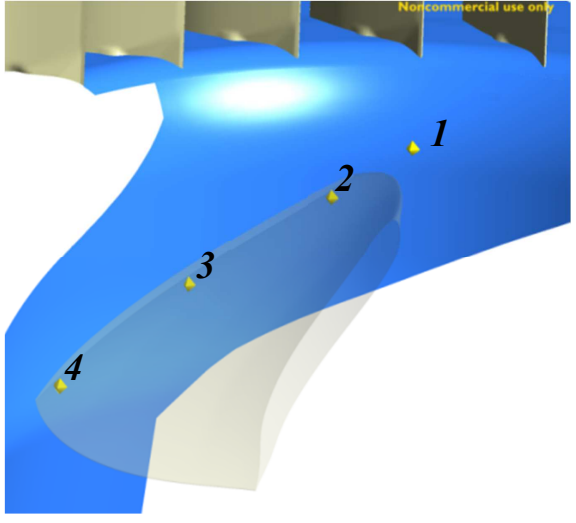

(b)

Fig. 10 Pressure points locations for the spanwise direction. (a) Suction side of the blade. (b) Pressure side.

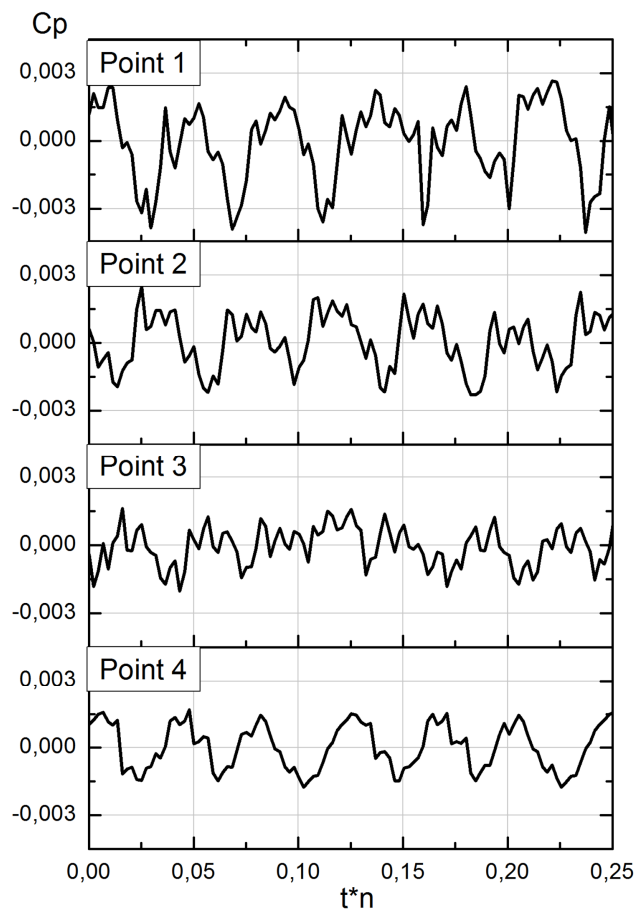

(a)

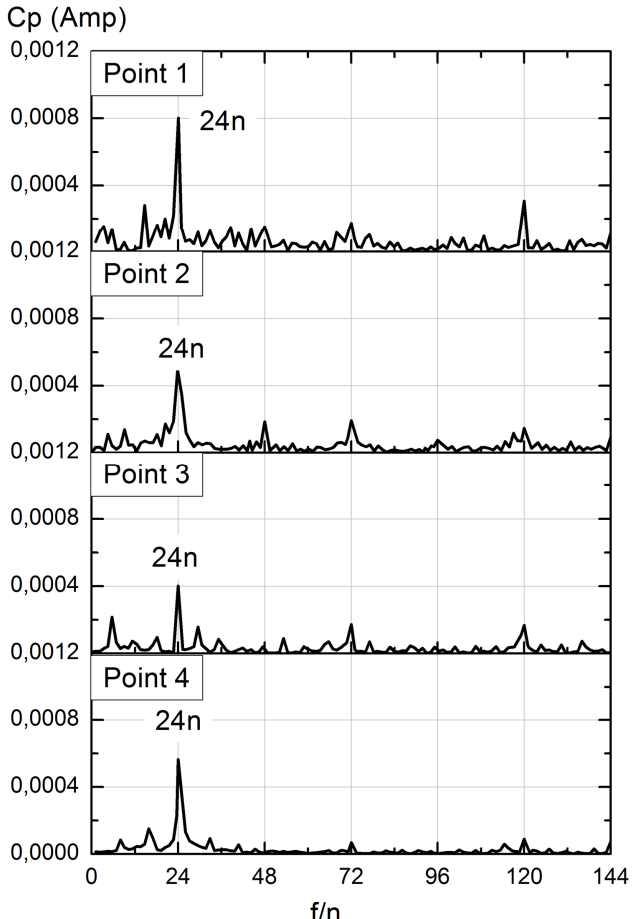

(b)

Fig. 11 Temporary signals (a) and spectrum (b) of pressure fluctuation over a spanwise direction. 


\section{Conclusions}

Prototype measurements at a large Kaplan turbine were used to validate the transient simulations performed using the software ANSYS CFX with the RNG k- $\varepsilon$ turbulence model. Good agreement was found in pressure pulsation signals at low frequencies, both in shape and amplitude. Rotor-Stator interactions frequencies can be captured with this scheme of simulation. Pressure points located through the spanwise direction show a fluctuation due to the runner blade passage across a nonuniform flow initiated at the wicket gate outlet. The frequency obtained in the rotational reference frame is 24 times the runner velocity, which is perceptible even closer to the outlet section. This would suggest that the existence of erosion patches at the discharge ring in concordance with the number of wicket gates, as could be verified at the prototype machine. The periodic pressure distribution circumferentially over the discharge ring is clearly related with this phenomenon.

Secondary flows, like the vortex generated due to wicket gate overhang and the tip gap vortex development were represented. The frequency of 25 times the runner velocity measured in prototype was not captured at CFD simulations. More research related with mesh configurations and usage of other turbulence models is required.

Next step in our research will be focus on the influence of the wicket gate overhang and its shape on the rotor-stator interaction.

$\begin{array}{llllll}\text { 6. Nomenclature } & & & & \\ \mathrm{n} & {\left[\mathrm{rot} \cdot \mathrm{s}^{-1}\right]} & \text { Passage runner frequency. } & \Delta \mathrm{z} & {[\mathrm{m}]} & \text { Tail water level. } \\ \mathrm{p}_{\mathrm{abs}} & {[\mathrm{Pa}]} & \text { Absolute static pressure. } & \bar{p} & {[\mathrm{~Pa}]} & \text { Time average static pressure. } \\ \mathrm{p}_{\text {ref }} & {[\mathrm{Pa}]} & \text { Reference static pressure. } & \mathrm{E} & {\left[\mathrm{j} \cdot \mathrm{kg}^{-1}\right]} & \text { Specific energy. } \\ \mathrm{p}_{\mathrm{est}} & {[\mathrm{Pa}]} & \text { Relative static pressure. } & \mathrm{H} & {[\mathrm{m}]} & \text { Net head. } \\ \mathrm{g} & {\left[\mathrm{m} \cdot \mathrm{s}^{-2}\right]} & \text { Gravity acceleration. } & \mathrm{Q} & {\left[\mathrm{m}^{3} \cdot \mathrm{s}^{-1}\right]} & \text { Flow rate. } \\ \rho & {\left[\mathrm{kg} \cdot \mathrm{m}^{-3}\right]} & \text { Water density. } & \text { Pot } & {[\mathrm{w}]} & \text { Power output. } \\ \mathrm{Cp} & {[-]} & \text { Pressure coefficient. } & \mathrm{e} & {[\mathrm{m}]} & \text { Gap size. }\end{array}$

\section{References}

[1] Nennemann, B. Vu, T. C. and Farhat, M., 2005. "CFD prediction of unsteady wicket gate-runner interaction in Francis turbines: A new standard hydraulic design procedure”. HYDRO 2005 International Conference and Exhibition, Villach, Austria.

[2] Zobeiri, A. Kueny, J. Farhat, M. y Avellan, F., 2006. "Pump turbine rotor-stator interactions in generating mode: pressure fluctuations in distributor channel", 23rd IAHR symposium. IAHR, Yokohama.

[3] Shuhong, L. Jianqiang, M. Jie, S. Yulin, W., 2009 "Pressure pulsation prediction by 3D turbulent unsteady flow simulation through whole flow passage of Kaplan turbine", Engineering Computations, Vol. 26 Iss: 8, pp.1006 - 1025.

[4] Nennemann, B. and Vu, T. C., 2007 "Kaplan turbine blade and discharge ring cavitation prediction using unsteady CFD". 2nd IAHR international meeting of the workgroup on cavitation and dynamic problems in hydraulic machinery and systems. Timisoara, Romania.

[5] Gagnon, JM. and Deschênes, C., 2006 "Numerical simulation of a Rotor-Stator Unsteady Interaction in a Propeller Turbine" CFD, Toronto.

[6] Gagnon, JM. Ciocan, G.D. Deschênes, C. Iliescu, M., 2008 "Numerical and Experimental Investigation of Rotor-Stator Interactions in an Axial Turbine: Numerical Interface Assessments", ASME 2008 Fluids Engineering Division Summer Meeting, FEDSM2008-55183, Jacksonville, Florida.

[7] Nilsson, H. and Davidson, L., 2000 "A Numerical Comparison on Four Operating Conditions in a Kaplan Water Turbine, Focusing on Tip Clearance Flow", 20th IAHR Symposium, Charlottem North Carolina, U.S.A. 